International Journal of Pure and Applied Mathematics

Volume 114 No. 3 2017, 457-471

ISSN: 1311-8080 (printed version); ISSN: 1314-3395 (on-line version)

url: http://www.ijpam.eu

doi: 10.12732 /ijpam.v114i3.4

\title{
COLUMN MEAN VANISHING MATRICES
}

\author{
Myungsup $\mathrm{Kim}^{1}$, Do Y. Kwak ${ }^{2} \S$ \\ ${ }^{1}$ Tunitel Co, Kwan Pyong Dong \\ Yusung-Ku, Daejeon, 34017, KOREA \\ ${ }^{2}$ Department of Mathematical Sciences \\ Korea Advanced Institute of Science and Technology \\ Daejeon, 34141, KOREA
}

\begin{abstract}
In this paper, we study some properties of a special class of matrices having orthonormal columns. These matrices appear in some applications, especially in wireless communications. We study the column property and spectral decomposition. Using these properties, we suggest a new method of generating such matrices. For $N$ even, the new method gives rise to a matrix which is more efficient. Numerical examples to compare two methods are included.
\end{abstract}

AMS Subject Classification: 65 N15, 65N30, 35J60

Key Words: orthogonal columns, column mean vanishing property, SVD

\section{Introduction}

In this paper, we study some properties of a special class of rectangular matrices having orthonormal columns. These matrices appear in some applications, especially, in OFDM-wireless communications [2],[3],[7],[5]. In OFDM, the signals must not have large jumps near the boundary of frequencies. Otherwise, the spectrum spread would interfere with neighboring channels. This kind of matrices can be used to design a technology to carry multiple signals without interference between channels [6]. We will study some properties and

Received: November 15, 2016

Revised: $\quad$ April 25, 2017

Published: $\quad$ May 23, 2017

(C) 2017 Academic Publications, Ltd. url: www.acadpubl.eu

$\S_{\text {Correspondence author }}$ 
and a spectral decomposition of them. Using these properties, we suggest a new method of generating such matrices. We use techniques of singular value decomposition(SVD) and nearest orthogonal matrix generation [1],[8],[9]. Numerical examples to compare two methods are included. For $N$ even, the new method gives rise to a matrix which is more efficient.

\section{Generation of a Matrix Having Orthonormal, Column Mean Vanishing Property}

We start a notion about the matrices.

Definition 2.1. We say a matrix $A$ has a column mean vanishing (CMV) property if the sum of each column is zero.

Let $F$ and $F^{-1}$ be the matrix representation of Fast Fourier Transform(FFT) and inverse Fast Fourier Transform (IFFT): Given any $n \times 1$ vector $\mathbf{x}$, its FFT and IFFT are given by

$$
\hat{\mathbf{x}}=F \mathbf{x} \text { and } \check{\mathbf{x}}=F^{-1} \mathbf{x},
$$

where $F=\left(f_{j k}\right)$ and $F^{-1}=\left(f_{j k}^{\prime}\right)$ are

$$
f_{j k}=e^{-\theta_{j} k}, f_{j k}^{\prime}=\frac{1}{n} e^{\theta_{j} k}, \text { with } \theta_{j}=\frac{2 \pi i j}{n}, i=\sqrt{-1} .
$$

Let $L=n \gg m$ and $N \ll L M=N-1$. Using an initial $N \times(N-1)$ matrix, IFFT, zero padding, removing jumps, and truncation, FFT, we will generate a new matrix having the desired properties. The following scheme is suggested in $[6]$.

\section{Algorithm Orth-CMV}

1. Given a $N \times(N-1)$ initial matrix $K$ with orthonormal columns.

2. Multiply by $n \times N$ matrix $\mathbf{P}$ obtaining $A=\mathbf{P} K$.

3. Perform IFFT to obtain $\mathbf{F}^{-1}(\mathbf{P} K)$.

4. Subtract the first row from all the rows, the result is $\mathbf{\Phi} \circ \mathbf{F}^{-1}(\mathbf{P} K)$.

5. Perform FFT to get $\mathbf{F} \circ \mathbf{\Phi} \circ \mathbf{F}^{-1}(\mathbf{P} K)$.

6. Multiply $\mathbf{P}^{T}$ to obtain $\hat{K}:=\mathbf{P}^{T} \circ \mathbf{F} \circ \mathbf{\Phi} \circ \mathbf{F}^{-1}(\mathbf{P} K)$.

7. Normalize each column of $\hat{K}$, call it by $\hat{K}_{0}$. 


\section{$\Theta$}

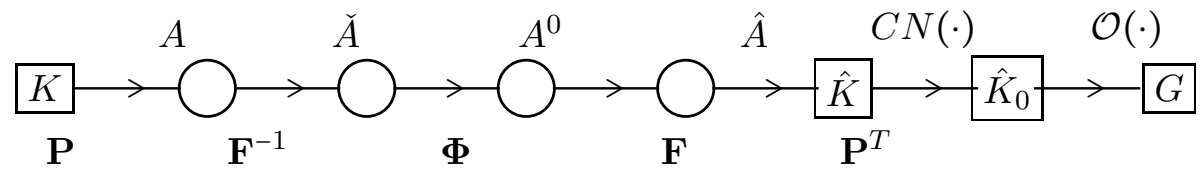

Figure 1: Signal flow diagram for matrix generation. $\boldsymbol{\Theta}$ is a jump removing operator in frequency domain, $C N(\cdot)$ and $\mathcal{O}(\cdot)$ are normalization and orthogonalization operator, resp.

8. Let $G=U V^{H}$ where $U \Sigma V^{H}$ is the SVD of $\hat{K}_{0}$.

In the next, we list some matrix notations:

\begin{tabular}{|l|rl|}
\hline Matrices & $K$ & Initial matrix to generate the matrix $G$ \\
& $A$ & Permuted and zero padded matrices of $K$ \\
$\check{A}$ & IFFT performed matrix of $A$ \\
& $A^{0}$ & Internal jump removed matrix \\
$\hat{A}$ & FFT performed matrix of $A$ \\
$\hat{K}$ & Jump removed matrix from $K$ \\
& $\hat{K}_{0}$ & Normalized matrix after jump removing \\
& $G$ & matrix with orthonormal columns \\
\hline Operators & $\mathbf{P}$ & Permutation and zero padding matrix \\
& $\mathbf{F}^{-1}$ & IFFT matrix \\
& $\mathbf{\Phi}$ & Jump removing matrix \\
& $\mathbf{F}$ & FFT matrix \\
& $\mathbf{P}^{T}$ & Permuting and truncating matrix \\
& $\boldsymbol{\Theta}$ & Jump removing matrix in frequency domain \\
\hline
\end{tabular}

Now we will explain more details of the algorithm: We first assume $N=$ $2 m+1$. Let the initial matrix $K$ of size $N \times(N-1)$ be given by

$$
K=\frac{1}{\sqrt{2}}\left[\begin{array}{cccc|ccc}
0 & 0 & 0 & 0 & 0 & 1 & 1 \\
\vdots & & & & . \cdot & 0 & 0 \\
\hline 0 & 0 & 1 & 1 & \cdots & 0 & 0 \\
1 & 1 & 0 & 0 & \cdots & 0 & 0 \\
0 & 0 & 0 & 0 & \cdots & 0 & 0 \\
1 & -1 & 0 & 0 & \cdots & 0 & 0 \\
0 & 0 & 1 & -1 & \cdots & 0 & 0 \\
\hline \vdots & & & & \ddots & 0 & 0 \\
0 & 0 & 0 & 0 & 0 & 1 & -1
\end{array}\right]
$$




\section{Step (2). Permute and Pad Zeros}

Starting from $K$, we construct an $L \times M$ matrix as follows: Move the last $m+1$ rows of $K$ to the first $m+1$ rows of $K$. Next fill it with pad with $L-M$ zero rows (called zero padding). This process can be expressed as $\mathbf{P} K$ where

$$
\mathbf{P}=\left[\begin{array}{c|c}
\underline{0}_{(m+1) \times m} & \mathbf{I}_{m+1} \\
\hline 0 & 0 \\
\vdots & \vdots \\
0 & 0 \\
\hline \mathbf{I}_{m} & \underline{0}_{m \times(m+1)}
\end{array}\right]
$$

Here $\mathbf{I}_{m}, \mathbf{I}_{m+1}$ are identity matrices of size $m$ and $m+1$.

\section{Steps (3) and (4) : IFFT Followed by Subtraction of the First Row}

Let us use the notation $K=\left(k_{i j}\right)$ and $K_{1}=\left(k_{i j}^{1}\right):=\mathbf{P} K$. Let $\check{K}_{1}=\mathbf{F}^{-1}(\mathbf{P} K)$ be the inverse FFT of $\mathbf{P} K$. By definition of IFFT (2.1), the first row of $\check{K}_{1}$ is

$$
\check{\mathbf{k}}_{1}=\left[\check{k}_{11}, \check{k}_{12}, \cdots, \check{k}_{1 M}\right]=\frac{1}{n}\left[\sum_{i=0}^{n-1} k_{i 1}^{1}, \sum_{i=0}^{n-1} k_{i 2}^{1}, \cdots, \sum_{i=0}^{n-1} k_{i M}^{1}\right] .
$$

The process of IFFT of permuting the rows and eliminating first row is described by

$$
\check{K}_{1}{ }^{\prime}=\left[\begin{array}{cccc}
\check{k}_{11} & \check{k}_{12} & \cdots & \check{k}_{1 M} \\
\check{k}_{21} & \check{k}_{22} & \cdots & \check{k}_{2 M} \\
\vdots & \vdots & \cdots & \vdots \\
\check{k}_{n, 1} & \check{k}_{n, 2} & \cdots & \check{k}_{n, M}
\end{array}\right]-\left[\begin{array}{cccc}
\check{k}_{11} & \check{k}_{12} & \cdots & \check{k}_{1 M} \\
\check{k}_{11} & \check{k}_{12} & \cdots & \check{k}_{1 M} \\
\vdots & \vdots & \cdots & \vdots \\
\check{k}_{11} & \check{k}_{12} & \cdots & \check{k}_{1 M}
\end{array}\right] \equiv \check{K}_{1}-\check{K}_{1}^{*} .
$$

Here $\check{K}_{1}^{*}$ is the matrix all of whose rows are the vector $\check{\mathbf{k}}_{1}$. Let $\boldsymbol{\Phi}$ be the operator involved in the elimination of first row in step (4) of the algorithm. Then

$$
\boldsymbol{\Phi} \equiv\left[\begin{array}{ccccc}
0 & 0 & 0 & \cdots & 0 \\
-1 & 1 & 0 & \cdots & 0 \\
-1 & 0 & 1 & \cdots & 0 \\
\vdots & \vdots & \vdots & \vdots & 0 \\
-1 & 0 & 0 & \cdots & 0 \\
-1 & 0 & 0 & \cdots & 1
\end{array}\right] .
$$




\section{Step (5) and (6). FFT, Truncation and Band Limit}

The step (5) is FFT and the process in step (6) corresponds to the permutation and truncation.

Lemma 2.1. The result of step (5) is

$$
\mathbf{F} \circ \mathbf{\Phi} \circ \mathbf{F}^{-1}(\mathbf{P} K)=\mathbf{P} K-\mathbf{F}\left(\check{K}_{1}^{*}\right) .
$$

Hence after step (6) we obtain the matrix

$$
\hat{K}:=K-\mathbf{P}^{T} \circ \mathbf{F}\left(\check{K}_{1}^{*}\right) .
$$

Proof. From (2.6) we see

$$
\begin{aligned}
\mathbf{F} \circ \mathbf{\Phi} \circ \mathbf{F}^{-1}(\mathbf{P} K) & =\mathbf{F} \circ F^{-1}(\mathbf{P} K)-\mathbf{F}\left(\check{K}_{1}^{*}\right) \\
& =\mathbf{P} K-\mathbf{F}\left(\check{K}_{1}^{*}\right),
\end{aligned}
$$

and hence

$$
\begin{aligned}
\mathbf{P}^{T} \circ \mathbf{F} \circ \boldsymbol{\Phi} \circ F^{-1}(\mathbf{P} K) & =\mathbf{P}^{T} \mathbf{P} K-\mathbf{P}^{T} \circ \mathbf{F}\left(\check{K}_{1}^{*}\right) \\
& =K-\mathbf{P}^{T} \circ \mathbf{F}\left(\check{K}_{1}^{*}\right):=\hat{K} .
\end{aligned}
$$

Now we compute the matrix $\mathbf{P}^{T} \circ F\left(\check{K}_{1}^{*}\right)$. Since the FFT of the vector $[1, \cdots, 1]^{T}$ is $[n, 0, \cdots, 0]^{T}$, we see the FFT of $\check{K}_{1}^{*}$

$$
\mathbf{F}\left(K_{1}^{*}\right)=n\left[\begin{array}{cccc}
\check{k}_{11} & \check{k}_{12} & \cdots & \check{k}_{1 M} \\
0 & 0 & 0 & 0 \\
\vdots & \vdots & \vdots & \vdots \\
0 & 0 & 0 & 0
\end{array}\right] .
$$

Here the first row is

$$
n\left[\check{k}_{11}, \cdots, \check{k}_{1 M}\right]=\left[\sum_{i=1}^{n} k_{i 1}, \cdots, \sum_{i=1}^{n} k_{i M}\right] \equiv\left[x_{11}, x_{12}, \cdots, x_{1 M}\right] \equiv \mathbf{x} .
$$

By multiplying by $\mathbf{P}^{T}$, we obtain $N \times M$ matrix

$$
\mathbf{P}^{T} \circ \mathbf{F}\left(\check{K}_{1}^{*}\right)=\left[\begin{array}{cccc}
0 & 0 & \cdots & 0 \\
\vdots & \vdots & \cdots & \vdots \\
-x_{11} & -x_{12} & \cdots & -x_{1 M} \\
\vdots & \vdots & \cdots & \vdots \\
0 & 0 & \cdots & 0
\end{array}\right]
$$


Now as the result of step (6), we obtain

$$
\hat{K}=\left[\begin{array}{cccc}
k_{11} & k_{12} & \cdots & k_{1 M} \\
\vdots & \vdots & \cdots & \vdots \\
k_{m+1,1}-x_{11} & k_{m+1,2}-x_{12} & \cdots & k_{m+1, M}-x_{1 M} \\
\vdots & \vdots & \cdots & \vdots \\
k_{N 1} & k_{N 2} & \cdots & k_{N M}
\end{array}\right]=K-\mathbf{P}^{T} \circ F\left(\check{K}_{1}^{*}\right)
$$

Lemma 2.2. The sum of all entries of each column of the matrix $\hat{K}$ or $\hat{K}_{0}$ is zero.

Proof. Clear from (2.10) and (2.12).

\section{Nearest Orthogonal Matrix}

The step (8) can be described by another way: Let

$$
\hat{K}_{0}=U \Sigma V^{H}
$$

be the singular value decomposition (SVD) of $\hat{K}_{0}$. Then the matrix $G=U V^{H}$ is the same as the polar decomposition [4]:

Lemma 2.3.

$$
U V^{H}=\hat{K}_{0}\left(\hat{K}_{0}^{H} \hat{K}_{0}\right)^{-1 / 2}
$$

It is also well-known that $G$ is the nearest matrix to $\hat{K}_{0}$ having orthonormal columns. (Corollary 2.3 of [4] and the remark following it.)

Theorem 2.1. The matrix $G$ obtained in step (8) satisfies CMV property:

Proof. Let $\overrightarrow{\mathbf{1}}=[1, \cdots, 1]$. Then by Lemma 2.2 , we have

$$
\overrightarrow{\mathbf{1}} \cdot \hat{K}_{0}=[0,0, \cdots, 0] .
$$

Hence by (2.14) we see

$$
\overrightarrow{\mathbf{1}} \cdot G=\overrightarrow{\mathbf{1}} \cdot \hat{K}_{0}\left(\hat{K}_{0}^{H} \hat{K}_{0}\right)^{-1 / 2}=[0,0, \cdots, 0] .
$$

\subsection{The Case $N$ Even}

Now we investigate the even case. 


\section{Steps (1), (2)}

The initial matrix is different from the odd case. Let $N=2 m$ and let the initial matrix $K$ of size $N \times(N-1)$ be given by

$$
K_{e}^{(0)}=\frac{1}{\sqrt{2}}\left[\begin{array}{cccc|cc}
0 & 0 & 0 & 0 & 1 & 1 \\
\vdots & & & . \cdot & 0 & 0 \\
\hline 0 & 1 & 1 & \cdots & 0 & 0 \\
1 & 0 & 0 & \cdots & 0 & 0 \\
-1 & 0 & 0 & \cdots & 0 & 0 \\
0 & 1 & -1 & \cdots & 0 & 0 \\
\hline \vdots & & & \ddots & 0 & 0 \\
0 & 0 & 0 & 0 & 1 & -1
\end{array}\right]
$$

This is obtained by removing the central row and first column from the odd case (2.3). Using this matrix we will generate a matrix having the desired properties. Starting from $K_{e}^{(0)}$, we proceed similarly to the odd case. The matrix $\mathbf{P}$ (resp. $\mathbf{P}^{T}$ ) involved in zero padding (resp. truncation) is the following

$$
\mathbf{P}=\left[\begin{array}{c|c}
\underline{0}_{m \times m} & \mathbf{I}_{m} \\
\hline 0 & 0 \\
\vdots & \vdots \\
0 & 0 \\
\hline \mathbf{I}_{m} & \underline{0}_{m \times m}
\end{array}\right], \quad \mathbf{P}^{T}=\left[\begin{array}{c|ccc|c}
\underline{0}_{m \times m} & 0 & \cdots & 0 & \mathbf{I}_{m} \\
& & & & \\
\hline \mathbf{I}_{m} & 0 & \cdots & 0 & \underline{0}_{m \times m}
\end{array}\right]
$$

Steps (3) through (8) are the Same

\section{Some Spectral Analysis - Property of Even (Odd) Columns}

Let $\mathbf{k}_{i}$ and $\mathbf{g}_{i}$ and denote the $i$-th column of the matrix $K$ and $G$ respectively.

Lemma 3.1. Then we have the following.

1. For odd $N$, the even columns of the matrix $K$ are the same as those of $K$

2. For even $N$, the odd columns of the matrix $K$ are the same as those of $K$. 
For example, if $N=5$, then

$$
\mathbf{k}_{2}=\frac{1}{\sqrt{2}}\left[\begin{array}{c}
0 \\
1 \\
0 \\
-1 \\
0
\end{array}\right]=\mathbf{g}_{2}, \quad \mathbf{k}_{4}=\frac{1}{\sqrt{2}}\left[\begin{array}{c}
1 \\
0 \\
0 \\
0 \\
-1
\end{array}\right]=\mathbf{g}_{4} .
$$

Proof. We explain the case when $N=5$, the general case is exactly the same. We see from (2.12) that $\hat{K}$ in step (6) has changed only in the third row. In fact, $\left[\begin{array}{llll}x_{11} & x_{12} & x_{13} & x_{14}\end{array}\right]=\frac{1}{\sqrt{2}}\left[\begin{array}{llll}2 & 0 & 2 & 0\end{array}\right]$. Hence we have

$$
\hat{K}=\left[\begin{array}{cccc}
k_{11} & k_{12} & k_{13} & k_{14} \\
k_{21} & k_{22} & k_{23} & k_{24} \\
k_{31}-x_{11} & k_{32} & k_{33}-x_{13} & k_{34} \\
k_{41} & k_{42} & k_{43} & k_{44} \\
k_{51} & k_{52} & k_{53} & k_{54}
\end{array}\right]
$$

Hence the even columns of $\hat{K}$ or the normalized matrix $\hat{K}_{0}$ are equal to the corresponding even columns of $K$.

Lemma 3.2. Then we have the following.

1. Let $N$ be odd. The even columns of $\hat{K}_{0}$ are orthogonal to all other columns of $\hat{K}_{0}$. As a consequence, for all even $j$, $\mathbf{e}_{j}$ is an eigenvector of $\hat{K}_{0}^{H} \hat{K}_{0}$ corresponding to eigenvalue 1.

2. Let $N$ be even. The odd columns of $\hat{K}_{0}$ are orthogonal to all other columns of $\hat{K}_{0}$. As a consequence, for all odd $j, \mathbf{e}_{j}$ is an eigenvector of $\hat{K}_{0}^{H} \hat{K}_{0}$ corresponding to eigenvalue 1 .

Proof. Assume $N$ is odd. The proof of even case is exactly the same. Let $\hat{\mathbf{k}}_{0, i}$ be the $i$-th column of $\hat{K}_{0}$. Then $\hat{\mathbf{k}}_{0, i}=\mathbf{k}_{i}$ for $i$ even and $\hat{\mathbf{k}}_{0, i}=\mathbf{k}_{i}-\mathbf{x}$ for $i$ odd. Since the $m+1$-st entry of even columns is zero, the subtraction of $\mathbf{x}$ from the third row in (3.1) does not affect the orthogonality. Hence when $j$ is even

$$
\hat{\mathbf{k}}_{0, i}^{T} \cdot \hat{\mathbf{k}}_{0, j}=\left\{\begin{array}{ll}
\left(\mathbf{k}_{i}-\mathbf{x}\right)^{T} \cdot \mathbf{k}_{j}=\delta_{i j} & \text { if } i \text { is odd } \\
\mathbf{k}_{i}^{T} \cdot \mathbf{k}_{j}=\delta_{i j} & \text { if } i \text { is even }
\end{array} .\right.
$$

Hence the $j$-th column of $\hat{K}_{0}^{H} \hat{K}_{0}$ satisfies

$$
\hat{K}_{0}^{H} \hat{K}_{0} \mathbf{e}_{j}=\hat{K}_{0}^{H} \hat{\mathbf{k}}_{0, j}=\left[\begin{array}{c}
\hat{\mathbf{k}}_{0,1}^{T} \cdot \hat{\mathbf{k}}_{0, j} \\
\hat{\mathbf{k}}_{0,2}^{T} \cdot \hat{\mathbf{k}}_{0, j} \\
\vdots \\
\hat{\mathbf{k}}_{0, N}^{T} \cdot \hat{\mathbf{k}}_{0, j}
\end{array}\right]=\mathbf{e}_{j} .
$$


This means that when $j$ is even, the $j$-th columns of $\hat{K}_{0}$ are orthogonal to all other columns of $\hat{K}_{0}$. Clearly (3.2) implies the second assertion of the lemma.

Example 3.1. For $N=5$ we see

$$
\hat{K}_{0}^{H} \hat{K}_{0}=\frac{1}{2}\left[\begin{array}{ccccc}
0 & 1 & -1 & 1 & 0 \\
0 & 1 & 0 & -1 & 0 \\
1 & 0 & -1 & 0 & 1 \\
1 & 0 & 0 & 0 & -1
\end{array}\right]\left[\begin{array}{cccc}
0 & 0 & 1 & 1 \\
1 & 1 & 0 & 0 \\
-1 & 0 & -1 & 0 \\
1 & -1 & 0 & 0 \\
0 & 0 & 1 & -1
\end{array}\right]=\frac{1}{2}\left[\begin{array}{cccc}
3 & |0| & 1 & |0| \\
0 & |2| & 0 & |0| \\
1 & |0| & 3 & |0| \\
0 & |0| & 0 & |2|
\end{array}\right]
$$

The zeros in the box keep the even columns of $\hat{K}_{0}$ orthogonal to other columns. In view of $(3.2), \hat{K}_{0}^{H} \hat{K}_{0}$ has two eigenvectors $\mathbf{e}_{j}, j=2,4$ corresponding to the eigenvalue 1 .

Theorem 3.2. We have the following result.

1. For $N$ odd, $G$ has eigenvector $\mathbf{e}_{j}$ for all $j$ even with the corresponding eigenvalue 1 . The even columns of $G=\hat{K}_{0}\left(\hat{K}_{0}^{H} \hat{K}_{0}\right)^{-1 / 2}$ are the same as those of $K$.

2. For $N$ even, $G$ has eigenvector $\mathbf{e}_{j}$ for all $j$ odd with the corresponding eigenvalue 1. The odd columns of $G=\hat{K}_{0}\left(\hat{K}_{0}^{H} \hat{K}_{0}\right)^{-1 / 2}$ are the same as those of $K$.

Proof. Since $\hat{K}_{0}=U \Sigma V^{H}$ from (2.13), we have the spectral decomposition of $\hat{K}_{0}^{H} \hat{K}_{0}$ :

$$
\hat{K}_{0}^{H} \hat{K}_{0}=V \Sigma^{H} \Sigma V^{H}:=V \Lambda V^{H}\left(V^{H}=V^{-1}\right),
$$

where by (3.2) $\Lambda$ and $V$ have the following form:

$$
\left.\Lambda=\left[\begin{array}{ccccc}
\lambda_{1} & 0 & 0 & \cdots & 0 \\
0 & 1 & 0 & \cdots & 0 \\
0 & 0 & \lambda_{3} & & 0 \\
\vdots & & \vdots & \ddots & 0 \\
0 & 0 & 0 & \cdots & 1
\end{array}\right],(N \text { odd }) \Lambda=\left[\begin{array}{ccccc}
1 & 0 & 0 & \cdots & 0 \\
0 & \lambda_{1} & 0 & \cdots & 0 \\
0 & 0 & 1 & & 0 \\
\vdots & & \vdots & \ddots & 0 \\
0 & 0 & 0 & \cdots & 1
\end{array}\right] \text { ( } N \text { even }\right)
$$

The eigenvector corresponding to the eigenvalue 1 is $\mathbf{e}_{j}$. Hence when $N$ is odd, $V \mathbf{e}_{j}=\mathbf{e}_{j}$ for $j$ even and so $V^{-1} \mathbf{e}_{j}=V^{-1} V \mathbf{e}_{j}=\mathbf{e}_{j}$. Hence for each even $j$,

$$
\begin{aligned}
\hat{K}_{0}\left(\hat{K}_{0}^{H} \hat{K}_{0}\right)^{-1 / 2} \mathbf{e}_{j} & =\hat{K}_{0} V \Lambda^{-1 / 2} V^{-1} \mathbf{e}_{j} \\
& =\hat{K}_{0} V \Lambda^{-1 / 2} \mathbf{e}_{j}
\end{aligned}
$$




$$
\begin{aligned}
& =\hat{K}_{0} V \mathbf{e}_{j} \\
& =\hat{K}_{0} \mathbf{e}_{j} .
\end{aligned}
$$

In view of (3.1), this is the same as $j$-th column of $K$ (normalization does not change even columns). While when $N$ is even, the same conclusion holds for $j$ odd.

\section{Numerical Example}

In all of the computations, we used the Matlab.

Example 4.1. When $N=5$ and $M=4$, the initial matrix is

$$
K=\frac{1}{\sqrt{2}}\left[\begin{array}{cccc}
0 & 0 & 1 & 1 \\
1 & 1 & 0 & 0 \\
0 & 0 & 0 & 0 \\
1 & -1 & 0 & 0 \\
0 & 0 & 1 & -1
\end{array}\right]
$$

With this we get

$$
G=\left[\begin{array}{cccc}
-0.1954 & -0.0000 & 0.5117 & 0.7071 \\
0.5117 & 0.7071 & -0.1954 & -0.0000 \\
-0.6325 & -0.0000 & -0.6325 & 0.0000 \\
0.5117 & -0.7071 & -0.1954 & -0.0000 \\
-0.1954 & 0.0000 & 0.5117 & -0.7071
\end{array}\right]
$$

Example 4.2. When $N=7, M=6$, we get

$$
G=\left[\begin{array}{cccccc}
-0.1466 & -0.0000 & -0.1466 & -0.0000 & 0.5605 & 0.7071 \\
-0.1466 & -0.0000 & 0.5605 & 0.7071 & -0.1466 & 0.0000 \\
0.5605 & 0.7071 & -0.1466 & 0.0000 & -0.1466 & 0.0000 \\
-0.5345 & 0.0000 & -0.5345 & -0.0000 & -0.5345 & 0.0000 \\
0.5605 & -0.7071 & -0.1466 & 0.0000 & -0.1466 & 0.0000 \\
-0.1466 & 0.0000 & 0.5605 & -0.7071 & -0.1466 & 0.0000 \\
-0.1466 & 0.0000 & -0.1466 & -0.0000 & 0.5605 & 0.7071
\end{array}\right]
$$

where the following initial matrix was used. 


$$
K=\left[\begin{array}{cccccc}
0 & 0 & 0 & 0 & 1 & 1 \\
0 & 0 & 1 & 1 & 0 & 0 \\
1 & 1 & 0 & 0 & 0 & 0 \\
0 & 0 & 0 & 0 & 0 & 0 \\
1 & -1 & 0 & 0 & 0 & 0 \\
0 & 0 & 1 & -1 & 0 & 0 \\
0 & 0 & 0 & 0 & 1 & -1
\end{array}\right]
$$

Example 4.3 ( $N$ even). When $N=6, M=5$, we get

$$
G=\left[\begin{array}{ccccc}
-0.1327 & -0.1723 & -0.0000 & 0.5348 & 0.7071 \\
-0.1327 & 0.5348 & 0.7071 & -0.1723 & 0.0000 \\
0.8934 & -0.1327 & 0.0000 & -0.1327 & -0.0000 \\
-0.3626 & -0.5924 & 0.0000 & -0.5924 & -0.0000 \\
-0.1327 & 0.5348 & -0.7071 & -0.1723 & 0.0000 \\
-0.1327 & -0.1723 & -0.0000 & 0.5348 & -0.7071
\end{array}\right]
$$

\section{A Direct Generation of $G$}

In this section we introduce a method of generating $G$ without using FFT and SVD. To do that, we first observe the following fact:

- If a matrix has a CMV property, then the Step (4) of the algorithm is not necessary.

Hence the operator $\boldsymbol{\Phi}$ in the Figure ?? becomes identity and we have

$$
\mathbf{P}^{T} \mathbf{F} \boldsymbol{\Phi} \mathbf{F}^{-1} \mathbf{P}=\mathbf{P}^{T} \mathbf{F} \mathbf{I}_{L} \mathbf{F}^{-1} \mathbf{P}=\mathbf{P}^{T} \mathbf{P}=\mathbf{I}_{N} .
$$

Here $\mathbf{I}_{L}$ and $\mathbf{I}_{N}$ are identity operators in $\mathbb{R}^{L \times L}$ and $\mathbb{R}^{N \times N}$ respectively. Hence the whole process reduces to finding the nearest orthogonal matrix only. (step (8)) Using this fact, we suggest a simple method to generate such a matrix. The minimal requirements are

1. Every column of the matrix $G$ is a unit vector.

2. All the columns of the matrix $G$ are orthogonal to each other

3. The sum of each columns of the matrix $G$ is zero. 
So we need at least three variables to design a matrix. In fact, three variables are enough for $N$ odd. For even $N$, it seems four variables are needed. We use an example to explain. Let $N=5, M=4$. From Theorem 3.2, we know the even columns of $G$ are the same as those of $K$. We set

$$
G=\left[\begin{array}{cccc}
c & 0 & b & \frac{1}{\sqrt{2}} \\
b & \frac{1}{\sqrt{2}} & c & 0 \\
a & 0 & a & 0 \\
b & -\frac{1}{\sqrt{2}} & c & 0 \\
c & 0 & b & -\frac{1}{\sqrt{2}}
\end{array}\right]
$$

and impose the orthonormality and CMV condition:

$$
\begin{array}{r}
a^{2}+2\left(b^{2}+c^{2}\right)=1 \\
a^{2}+4 b c=0 \\
a+2 b+2 c=0 .
\end{array}
$$

Solving (5.1)-(5.2) we get $2 c^{2}+2 b^{2}-4 c b=2(b-c)^{2}=1$ and together with (5.3) we get

$$
a=-0.6325, b=0.5117, c=-0.1954 .
$$

These values gives the same $G$ as Example 4.1. The solution is not unique and we see another solution

$$
a=-0.5345, b=0.5345, c=-0.2673 \text {. }
$$

Example 5.1. For $N=7$ we assume the matrix of the following form:

$$
G=\left[\begin{array}{cccccc}
c & 0.0 & c & 0.0 & b & \frac{1}{\sqrt{2}} \\
c & 0.0 & b & \frac{1}{\sqrt{2}} & c & 0.0 \\
b & \frac{1}{\sqrt{2}} & c & 0.0 & c & 0.0 \\
a & 0.0 & a & 0.0 & a & 0.0 \\
b & -\frac{1}{\sqrt{2}} & c & 0.0 & c & 0.0 \\
c & 0.0 & b & -\frac{1}{\sqrt{2}} & c & 0.0 \\
c & 0.0 & c & 0.0 & b & -\frac{1}{\sqrt{2}}
\end{array}\right]
$$

We impose orthonormality conditions and CMV condition as before, to have

$$
\begin{aligned}
a^{2}+2\left(b^{2}+2 c^{2}\right) & =1 \\
a^{2}+4 b c+2 c^{2} & =0
\end{aligned}
$$




$$
a+2 b+4 c=0
$$

Solving this algebraic system we get the following values

$$
a=-0.5345, b=0.5605, c=-0.1466 .
$$

The corresponding matrix is

$$
G=\left[\begin{array}{ccccc}
-0.1466 & -0.0000 & -0.1466 & -0.0000 & 0.5605 \\
-0.1466 & -0.0000 & 0.5605 & 0.7071 & -0.1466 \\
0.5605 & 0.7071 & -0.1466 & 0.0000 & -0.1466 \\
-0.5345 & 0.0000 & -0.5345 & -0.0000 & -0.5345 \\
0.5605 & -0.7071 & -0.1466 & 0.0000 & -0.1466 \\
-0.1466 & 0.0000 & 0.5605 & -0.7071 & -0.1466 \\
-0.1466 & 0.0000 & -0.1466 & -0.0000 & 0.5605
\end{array}\right]
$$

which is the same as Example 4.2. Another solution is

$$
a=0.5345, b=0.3823, c=-0.3248 .
$$

More generally, we can construct any size of $K$ by assuming the even columns are

$$
\begin{aligned}
& {[0,0, \cdots, 0, \sqrt{2} / 2,0,-\sqrt{2} / 2,0,0, \cdots, 0]^{T},} \\
& {[0, \cdots, 0, \sqrt{2} / 2,0,0,0,-\sqrt{2} / 2,0, \cdots, 0]^{T},} \\
& \cdots \cdots \cdots \\
& {[\sqrt{2} / 2,0,0, \cdots, 0,0,0,0, \cdots, 0,-\sqrt{2} / 2]^{T}}
\end{aligned}
$$

while the odd columns are of the form

$$
\begin{aligned}
& {[c, c, \cdots, c, c, b, a, b, c, c, \cdots, c, c]^{T},} \\
& {[c, c, c, \cdots, b, c, a, c, b, c, \cdots, c, c]^{T},} \\
& {[c, c, \cdots, b, c, c, a, c, c, b, \cdots, c, c]^{T}} \\
& \cdots \cdots \cdots \\
& {[b, c, c, c, \cdots, c, c, a, c, c, \cdots, c, b]^{T}}
\end{aligned}
$$

Now impose the following conditions: for $j=2,3, \cdots$,

$$
\begin{aligned}
a^{2}+2\left(b^{2}+(j-1) c^{2}\right) & =1 \\
a^{2}+4 b c+2(j-2) c^{2} & =0 \\
a+2 b+2(j-1) c & =0 .
\end{aligned}
$$

By solving this simple algebraic system by Newton's method with certain initial values, we can find a desired matrix $G$ of any size. 
Example 5.2. $[9 \times 9]$ Assume

$$
G=\left[\begin{array}{cccccccc}
c & 0 & c & 0 & c & 0 & b & \frac{1}{\sqrt{2}} \\
c & 0 & c & 0 & b & \frac{1}{\sqrt{2}} & c & 0 \\
c & 0 & b & \frac{1}{\sqrt{2}} & c & 0 & c & 0 \\
b & \frac{1}{\sqrt{2}} & c & 0 & c & 0 & c & 0 \\
a & 0 & a & 0 & a & 0 & a & 0 \\
b & -\frac{1}{\sqrt{2}} & c & 0 & c & 0 & c & 0 \\
c & 0 & b & -\frac{1}{\sqrt{2}} & c & 0 & c & 0 \\
c & 0 & c & 0 & b & -\frac{1}{\sqrt{2}} & c & 0 \\
c & 0 & c & 0 & c & 0 & b & -\frac{1}{\sqrt{2}}
\end{array}\right]
$$

We need to solve

$$
\begin{aligned}
a^{2}+2\left(b^{2}+3 c^{2}\right) & =1 \\
4 c^{2}+4 b c+a^{2} & =0 \\
a+2 b+6 c & =0 .
\end{aligned}
$$

For example, with initial guess $[a, b, c]=[-0.6,-0.7,0.3]$, we obtain

$$
a=-0.4714, b=-0.4714, c=0.2357 .
$$

But with different initial $[-0.6,40.7,0.3]$, we obtain

$$
a=-0.4714, b=0.5893, c=-0.1179 .
$$

Example 5.3. $[11 \times 11]$ Let

$$
G=\left[\begin{array}{cccccccccc}
c & -0.0 & c & -0.0 & c & -0.0 & c & -0.0 & b & \frac{1}{\sqrt{2}} \\
c & -0.0 & c & -0.0 & c & -0.0 & b & \frac{1}{\sqrt{2}} & c & 0.0 \\
c & -0.0 & c & -0.0 & b & \frac{1}{\sqrt{2}} & c & -0.0 & c & 0.0 \\
c & -0.0 & b & \frac{1}{\sqrt{2}} & c & -0.0 & c & -0.0 & c & 0.0 \\
b & \frac{1}{\sqrt{2}} & c & 0.0 & c & 0.0 & c & -0.0 & c & 0.0 \\
a & 0.0 & a & 0.0 & a & 0.0 & a & 0.0 & a & 0.0 \\
b & -\frac{1}{\sqrt{2}} & c & 0.0 & c & 0.0 & c & -0.0 & c & 0.0 \\
c & 0.0 & b & -\frac{1}{\sqrt{2}} & c & 0.0 & c & 0.0 & c & 0.0 \\
c & 0.0 & c & 0.0 & b & -\frac{1}{\sqrt{2}} & c & 0.0 & c & 0.0 \\
c & -0.0 & c & -0.0 & c & -0.0 & b & -\frac{1}{\sqrt{2}} & c & 0.0 \\
c & -0.0 & c & -0.0 & c & -0.0 & c & -0.0 & b & -\frac{1}{\sqrt{2}}
\end{array}\right]
$$


For this case, we need to solve

$$
\begin{aligned}
a^{2}+2\left(b^{2}+4 c^{2}\right) & =1 \\
6 c^{2}+4 b c+a^{2} & =0 \\
a+2 b+8 c & =0 .
\end{aligned}
$$

With initial value $[-0.4264,0.6083,-0.0988]$, we get

$$
a=-0.4264, b=0.6083, c=-0.0988 .
$$

while with initial value $[1.0,0.3,-1.6]$, we get

$$
a=0.4264, b=0.5230, c=-0.1841 .
$$

\section{References}

[1] Banerjee, Sudipto, Roy, and Anindya, Linear Algebra and Matrix Analysis for Statistics, Texts in Statistical Science, Chapman and Hall/CRC, 2014.

[2] O. Edfors, M. Sandell, J. -J. van de Beek, and S. K. Wilson, OFDM channel estimation by singular value decomposition, IEEE Trans. Commun., vol. 46, pp. 931-939, July 1998.

[3] Fleming Bjerge Frederiksen and Ramjee Prasad, An Overview of OFDM and Related Techniques Towards Development of Future Wireless Multimedia Communications, in Radio and Wireless Conference, RAWCON 2002, pp19-22, 2002.

[4] Nicholas J. Higham, Computing the Polar Decomposition - With Applications, SIAM J. SCI. STAT. COMPUT. vol. 7, no. 4, Oct. 1986

[5] Myungsup Kim and Do Young Kwak, Generalized OFDM, Submitted to IEEE Communications Letters.

[6] Myungsup Kim and Do Young Kwak, Generalized OFDM for the 5th Generation Mobile Communications, Submitted to VCT2017.

[7] Raed Meslehz, Hany Elgalaz, and Harald Haas, An Overview of Indoor OFDM/DMT Optical Communication Systems Networks and Digital Signal Processing, in SNDSP, pp. 566-570, 2010.

[8] R.-C. Li., New Perturbation bounds for the polar factor, SIAM J. Matrix Anal. Appl. Vol. 16, No. 1, pp. 327-332, January 1995.

[9] Ji-Guang Sun, A Note on Backward Perturbations for the Hermitian Eigenvalue Problem, pp. 385-393 of BIT 35 (1995). 
